\title{
Hipertiroidismo y Síndrome de Down: Caso clínico
}

\author{
FRANCISCA EYZAGUIRRE C. ${ }^{1,2}$, NANCY UNANUE M. ${ }^{1}$, VERÓNICA MERICQ G. ${ }^{1}$ \\ 1. Instituto de Investigaciones Materno-Infantil, Universidad de Chile, Hospital San Borja Arriarán. \\ 2. Becada Sociedad Chilena de Endocrinología y Diabetes (SOCHED).
}

\begin{abstract}
Hyperthyroidism and Down Syndrome: a present diagnosis

A female patient with Down Syndrome and without cardiac defects. During infancy, she had low weight increment secondary to repeated hospital admissions due to obstructive respiratory tract episodes. In addition, she attends regularly to the gastroenterology clinic due to intermittent diarrhea. At the age of 9.4 years-old, she presented liquid stools 5-6 times/day, associated to a decrease of $7 \mathrm{~kg}$ in 5 months and marked hyperactivity. She is admitted with tachycardia, arterial hypertension and high liver enzymes $(\mathrm{SGOT}=63 \mathrm{U} / 1$ and $\mathrm{SGPT}=97 \mathrm{U} / 1)$. The ECG showed sinus tachycardia. She is discharged without etiological diagnosis. In the mean time, annual thyroid function requested for endocrinology control showed TSH $<0,1 \mathrm{uUI} / \mathrm{ml}, \mathrm{T} 3=482 \mathrm{ng} / \mathrm{dl}$ and total T4 $=15,4 \mathrm{ug} / \mathrm{dl}$, evidencing clear hyperthyroidism and beginning therapy with propylthiouracil $10 \mathrm{mg} / \mathrm{kg} /$ day and propanolol $1,3 \mathrm{mg} / \mathrm{kg} / \mathrm{day}$. After 3 weeks, the patient showed less hyperactivity, normal stools, normal sleep-awake cycle and recovered weight. By 6 weeks, thyroid hormones and transaminases were within normal ranges.
\end{abstract}

(Key words: Down's sybdrome, hyperthyroidism)

Rev Chil Pediatr 2008; 79 (3): 290-294

\section{RESUMEN}

Objetivo: Describir una asociación poco frecuente de Síndrome de Down con Hipertiroidismo. Caso Clinico: Paciente de sexo femenino, portadora de síndrome de Down, sin antecedentes de cardiopatía congénita. Evolucionó con mal incremento ponderal en el período de lactante, hospitalizaciones repetidas por cuadros respiratorios obstructivos. En control en gastroenterología por episodios de diarrea intermitente, y en genética en forma regular. Cuadro actual se inicia a los 9,4 años, caracterizado por deposiciones líquidas 5-6 veces al día, asociado a baja de peso aproximada de $7 \mathrm{~kg}$ en 5 meses e hiperactividad. Se hospitalizó para estudio y se pesquisaron cifras tensionales elevadas y taquicardia. En perfil bioquímico aparece SGOT 63 U/1 y SGTP 97 U/1. Electrocardiograma informa taquicardia sinusal. Alta sin etiología clara, se solicita función tiroidea: TSH $<0,1 \mathrm{uUI} / \mathrm{ml}$, T3 $482 \mathrm{ng} / \mathrm{dl}$, T4 total 15,4 ug/dl realizándose diagnóstico de hipertiroidismo. Inició terapia con propiltiouracilo $10 \mathrm{mg} / \mathrm{kg} /$ día y propanolol $1,3 \mathrm{mg} / \mathrm{kg} / \mathrm{día}$. A las 3 semanas de iniciado el tratamiento, la paciente presenta menor hiperactividad, deposiciones normales, 
regulación de la hiperactividad y ciclo sueño-vigilia, recuperando peso. A las 6 semanas, los niveles de T3, T4 y transaminasas eran normales. El hipertiroidismo se observa con mucha menor frecuencia que el hipotiroidismo en niños y adultos con síndrome de Down. En series numerosas de pacientes con trisomía 21, se describen en general un bajo porcentaje de casos de hipertiroidismo, dentro de los cuales se incluyen la enfermedad de Graves y la Hashitoxicosis

(Palabras clave: síndrome de Down, hipertiroidismo, hipertransaminasemia)

Rev Chil Pediatr 2008; 79 (3): 290-294

\section{Introducción}

La trisomía 21 es la cromosomopatía más frecuente, presentando en Chile una prevalencia de 2,35 por 1000 recién nacidos vivos, en un estudio realizado en la maternidad del hospital José Joaquín Aguirre ${ }^{1}$. Este grupo de pacientes presenta patologías asociadas tales como cardiopatía congénita (hasta un 30\% de los casos), retraso del desarrollo psicomotor, alteraciones hematológicas como reacción leucemoide en el período neonatal o franca leucemia (linfática, mieloide y megacariocítica aguda) a edades posteriores, atresia o estenosis duodenal, ano imperforado, enfermedad de Hirschprung, luxación de la articulación atlanto-axoidea y variadas patologías autoinmunes, entre otras ${ }^{2-5}$.

Dentro de las patologías autoinmunes que exhiben los niños con trisomía 21, se encuentra la diabetes mellitus tipo 1 (DM1), que generalmente en ellos se diagnostica a una edad más precoz $^{6}$ e incluso presentan anticuerpos antiislotes en mayor proporción comparado con controles, antes de desarrollar la DM1 ${ }^{7}$. Estos niños también presentan una mayor frecuencia de enfermedad celíaca, alopecia y desarrollo precoz de enfermedad de Alzheimer ${ }^{8}$.

Con respecto a la función tiroidea en este grupo de niños, se ha descrito una alta incidencia de alteraciones, entre las cuales predomina el hipotiroidismo. Los niños con trisomía 21 presentan una frecuencia de hipotiroidismo congénito entre 28 y 30 veces mayor que los recién nacidos sanos ${ }^{9}$. Asimismo, muchos de estos pacientes presentan niveles elevados de TSH, con niveles normales de T3 y T4; esta condición se denomina hipotiroidismo congénito compensado subclínico, probablemente secundario a una inmadurez en el eje hipotálamo-hipófisistiroides ${ }^{10}$. Dentro de las causas de hipotiroidismo a edades posteriores, destaca el hipotiroidismo subclínico (25-30\% de los pacientes), que puede hacerse manifiesto más tarde y en forma especial, aquel de origen autoinmune ${ }^{11}$. En la vida adulta, el hipotiroidismo puede alcanzar una prevalencia de hasta un $54 \% \%^{12}$.

Con respecto al hipertiroidismo, esta condición se observa con mucha menor frecuencia en niños y adultos con síndrome de Down. En series numerosas de pacientes con trisomía 21 , se describen en general un bajo porcentaje de casos de hipertiroidismo, dentro de los cuales se incluyen la enfermedad de Graves y la Hashitoxicosis ${ }^{13-15}$.

\section{Caso clínico}

Paciente de sexo femenino, producto de tercer embarazo controlado, sin patología. Nació por parto prematuro por cesárea a las 32 semanas, con un peso de 1550 gr (AEG), talla $41 \mathrm{~cm}$ y CC $28 \mathrm{~cm}$. Apgar 8-9. Hospitalizada en neonatología por 13 días para estudio por diagnóstico de síndrome de Down, sin cardiopatía (cariotipo 47, XX +21 en 35 mitosis).

Evolucionó con cuadros de reflujo gastroesofágico, bronquitis obstructiva recurrente y oxígeno-dependencia hasta los 6 meses. Hospitalizada 5 veces por cuadros respiratorios el primer año de vida. Se controla en policlínico de nutrición por mal incremento ponderal los primeros meses de vida y desde los 5 años de edad, en estudio por equipo de gastroenterología del Hospital Clínico San Borja arriarán por cuadros de diarrea intermitente. Se realizaron electrolitos del sudor que fueron normales y anticuerpos antiendomisio que resultaron negativos. Pese a sus episodios de diarrea, la curva de crecimiento era normal hasta los 9 años. 
En control en policlínico de genética y endocrino en forma regular, con función tiroidea anual normal hasta los 8,8 años. Evaluada por oftalmólogo, quien le indica lentes por diagnóstico de estrabismo y astigmatismo y evaluación por otorrinolaringólogo, normal.

El cuadro se inició a los 9,4 años de edad, caracterizado por deposiciones líquidas 5-6 veces al día, hiperactividad, alteración del ritmo de sueño y pérdida de peso aproximada de $7 \mathrm{~kg}$ en 5 meses, con apetito conservado. Se hospitalizó para estudio debido a la persistencia de la diarrea, y en ese momento se pesquisan cifras tensionales elevadas (PAM 69-83), con frecuencias cardíacas que oscilaban entre 91-148 latidos/minuto, afebril. En el perfil bioquímico aparece SGOT 63 U/1, SGPT 97 U/1 y FA 214 $\mathrm{U} / 1$. Se realizó ECG que informó taquicardia sinusal. Dentro del estudio etiológico del cuadro diarreico, se realizan coprocultivo y examen parasitológico que resultaron negativos.

La paciente se dió de alta sin etiología clara del cuadro y se derivó a policlínico de gastroen-

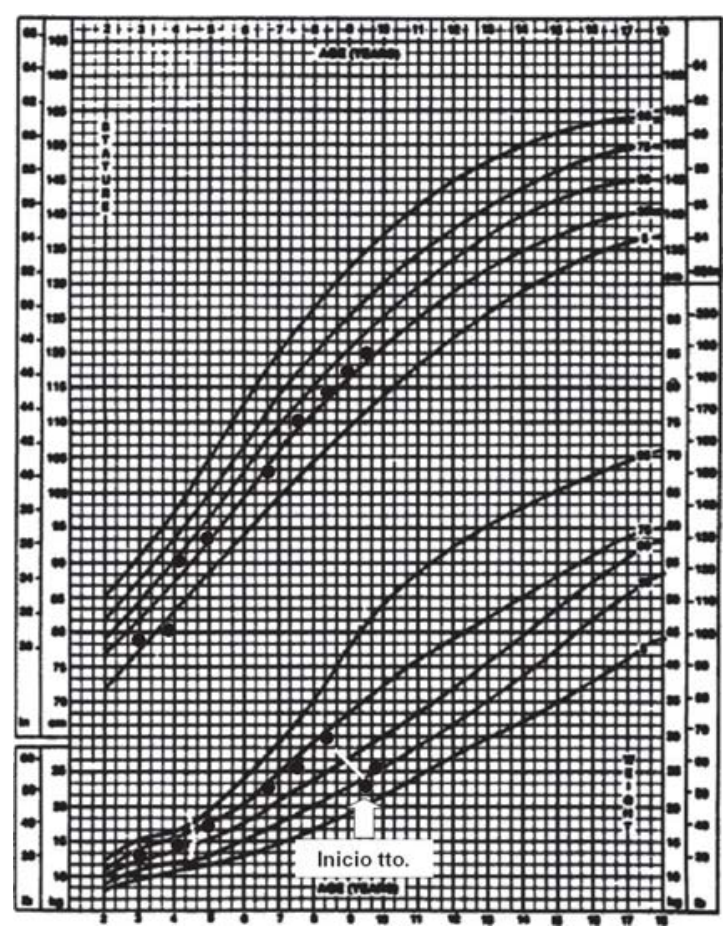

Figura 1. Curva de crecimiento para niñas con Síndrome de Down. terología donde se objetivó baja de peso (figura 1), y se evaluó el resultado de la función tiroidea (solicitada para su control anual en endocrino) compatible con hipertiroidismo: $\mathrm{TSH}<0,1 \mathrm{uUI} /$ $\mathrm{ml}, \mathrm{T} 3482 \mathrm{ng} / \mathrm{dl}$, T4 total 15,4 ug/dl. Se deriva a endocrinología para su manejo y dentro del estudio complementario se realiza una ecografia tiroidea en la que se describe bocio difuso, con aumento importante de la vascularización. Se miden niveles de anticuerpos anti-receptor de TSH (TRAb) que fueron 90,7\%, anticuerpos antimicrosomales 1:200 y anti-tiroglobulina, que fueron negativos (tabla 1). Al examen físico destacaba bocio palpable firme (aumento de tamaño 2-3 veces), taquicardia entre 90-100 latidos/minuto, sin exoftalmo ni temblor fino. Destacaba en la paciente una gran hiperactividad y sudoración de manos. En ese momento se inició terapia con propiltiouracilo $10 \mathrm{mg} / \mathrm{kg} /$ día y propanolol 1,3 mg/kg/día. A las 3 semanas de iniciado el tratamiento, la paciente presentaba menor hiperactividad y deposiciones normales 1-2 veces/día.

A las 6 semanas de terapia con propiltiouracilo, los niveles de T3, T4 y transaminasas eran normales, sin evidencias de efectos adversos asociados al tratamiento. La madre refiere que la paciente duerme tranquila toda la noche, se alimenta con buen apetito, ha recuperado peso, sus deposiciones son de consistencia normal y la hiperactividad ha disminuido en forma significativa. En la tabla 1 se puede observar la evolución de los exámenes de laboratorio. Al examen físico el bocio ha disminuido de tamaño, no presenta taquicardia ni sudoración aumentada.

Tabla 1. Resumen de la evolución de los exámenes de laboratorio

\begin{tabular}{llrrrr}
\hline Edad (años) & 7 & 8,8 & 9,4 & 9,6 & 9,8 \\
TSH (uUl/ml) & 0,7 & 3,0 & $<0,1$ & 0,1 & 0,1 \\
T4 (ug/dl) & 7,9 & & 15,4 & 16,1 & 5,1 \\
T4 libre (ng/dl) & & 1,8 & & 7,4 & 1,5 \\
T3 (ng/dl) & 93 & 482 & 136 & 143 \\
TRAb (\%) & & & 90,7 & \\
Antic antimicrosomales & & & $1: 200$ & \\
Antic antitiroglobulinas & & & $(-)$ & \\
SGOT/SGPT (U/l) & & $56 / 93$ & & $32 / 45$ \\
\hline
\end{tabular}




\section{Discusión}

Se presenta este caso clínico, dada la asociación poco habitual del síndrome de Down con hipertiroidismo. Sin embargo, debido a la elevada frecuencia de patología autoinmune en este grupo de pacientes, no sorprende que puedan presentar hipertiroidismo. Se ha asociado la enfermedad de Graves y el síndrome de Down con HLA BW46, siendo este genotipo poco constante en este grupo de pacientes ${ }^{13}$.

Llama la atención en el caso recientemente descrito, que los síntomas de hipertiroidismo fueron graduales, predominando el aumento del tránsito intestinal asociado a una significativa pérdida de peso. Como esta paciente tenía antecedentes de diarreas intermitentes de larga evolución, este síntoma pasó inadvertido inicialmente, lo que retrasó el diagnóstico. Posteriormente se asoció la irritabilidad, trastorno del sueño, hiperactividad y las cifras tensionales elevadas y taquicardia en el momento de su hospitalización. Esta evolución de 5 meses no es frecuente en niños con hipertiroidismo, pero se ha descrito una clínica más tenue en los pacientes con síndrome de Down, planteándose como posibilidad una resistencia periférica parcial a las hormonas tiroideas ${ }^{15}$.

El aumento de las transaminasas que muchas veces se observa en el hipertiroidismo, fue un factor muy importante en el diagnóstico de esta paciente. Esta hipertransaminasemia es multifactorial, siendo causada por un efecto directo de las hormonas tiroideas a nivel hepático, malnutrición, anoxia hepática, insuficiencia cardíaca congestiva (en casos que la presen$\tan )$ e infecciones asociadas, entre otras ${ }^{16}$.

Si bien esta paciente presentaba niveles muy elevados de TRAb, los anticuerpos antimicrosomales también eran positivos, lo que no nos permite descartar que este cuadro corresponda a una hashitoxicosis y no a una enfermedad de Graves. Antes de iniciar el tratamiento, no fue posible hacer un examen de captación de yodo, el que nos habría permitido apoyar con mayor certeza uno u otro diagnóstico.

En cuanto al tratamiento del hipertiroidismo en los pacientes con síndrome de Down, éste no difiere del utilizado en niños sin esta condición. En el caso de esta paciente, se optó por terapia con propiltiouracilo, dado su efecto a nivel tiroideo, inhibiendo la organificación del yodo, induciendo la apoptosis de los linfocitos que infiltran la glándula, y por otra parte, su efecto periférico inhibiendo la conversión de T4 a T3 $^{17}$. Si bien es un tratamiento que puede inducir efectos adversos severos, aunque poco frecuentes, es un medicamento de bajo costo y disponible más fácilmente que el metimazol, en el sistema público de salud. Se asoció a propanolol, (bloqueador $\beta$ ) dada la taquicardia y la elevación de la presión arterial. Clínicamente la respuesta fue bastante rápida, recuperando al mes de tratamiento, un ciclo de sueño-vigilia normal y la capacidad de realizar sus tareas habituales. La normalización de la función tiroidea ha sido un poco más lenta, en especial la recuperación de los niveles normales de TSH (tabla 1), pero la mejoría clínica ha sido significativa. Aún hace falta mantener el tratamiento hasta normalizar absolutamente los síntomas y los niveles de hormonas tiroideas. En niños, se podría esperar hasta dos años de terapia por vía oral con drogas antitiroideas (si es que no presenta efectos adversos graves), antes de plantear terapia con yodo radioactivo, cuyas dosis no están exactamente definidas ${ }^{18}$. La cirugía queda reservada para pacientes en los cuales no hay respuesta a tratamiento con fármacos orales y yodo radioactivo, situación muy poco frecuente en niños ${ }^{19}$.

Dado que una adecuada función tiroidea tiene gran importancia en el desarrollo antropométrico y psicomotor de los niños con trisomía 21, se hace necesario sugerir el control en forma anual en aquellos con buena evolución de su curva de crecimiento y de su desarrollo psicomotor, sin olvidar que este grupo de pacientes por la mayor frecuencia de patologías autoinmunes que presentan, pueden desarrollar hipertiroidismo.

\section{Referencias}

1.- Nazer J, Eaglin M, Cifuentes L: Incidencia del síndrome de Down en la maternidad del hospital Clínico de la Universidad de Chile. Un registro de 25 años: 19721997. Rev Méd Chile 1998; 126: 383-90.

2.- Korenberg Jr, Chen Xn, Schipper $R$, et al: Down syndrome phenotypes: the consequences of chromo- 
somal imbalance. Proc Nat Acad Sci 1994; 91: $4997-$ 5001.

3.- Korenberg J, Kawashima H, Pulst S, et al: Molecular definition of the region of chromosome 21 that causes features of the Down syndrome phenotype. Am J Hum Genet 1990; 47: 236-46.

4.- Henry E, Walter D, Wiedmeier SE, Christensen RD: Hematological abnormalities during the first week of life among neonates with Down syndrome: data from a multihospital healthcare system. Am J Med Genet 2007; 143a: 42-50.

5.- Korenberg J, Bradley C, Disteche C: Down syndrome: molecular mapping of congenital heart disease and duodenal stenosis. Am J Hum Genet 1992; 50: 294302.

6.- Shield JP, Wadsworth EJ, Hassold TJ, Judis LA, Jacobs $P A$ : Is disomic homozygosity at the apeced locus the cause of increased autoimmunity in Down's syndrome? Arch Dis Child 1999; 81(2): 147-50.

7.- Gillespie KM, Dix RJ, Williams AJ, et al: Islet autoimmunity in children with Down's syndrome. Diabetes 2006; 55 (11): 3185-8.

8.- Goate A, Chartier-Harllin MC, Mullan M, Brown J, Crawford F, et al: Segregation of a missense mutation in the amyloid precursor protein gene with familial Alzheimer's disease. Nature 1991; 349: 704-6.

9.- Fort P, Lifshitz F, Bellisario R, et al: Abnormalities of thyroid function in infants with Down syndrome. J Pediatr 1984; 104; 545-9.

10.- Cutler AT, Benerza-Obeiter R, Brink SJ: Thyroid function in young children with Down syndrome. Am
J Dis Child 1985; 140: 479-83.

11.- Tüysüz B, Beker DB: Thyroid dysfunction in children with Down's syndrome. Acta Paediatr 2001; 90: 138993.

12.- Rubello D, Pozzan GB, Casara D, et al: Natural course of subclinical hypothyroidism in Down's síndrome: prospective study results and therapeutic considerations. J Endocrinol Invest 1995; 17: 35-40.

13.- Soriano Guillén L, Muñoz Calvo MT, Pozo Román J, Martínez Pérez J, Baño Rodrigo A, Argente Oliver J: Enfermedad de Graves en pacientes con síndrome de Down. An Pediatr (Barc) 2003; 58 (1): 63-6.

14.- Gruñeiro DE Papendieck L, Chiesa A, Bastida MG, Alonso G, Finkielstein G, Heinrich JJ: Thyroid dysfunction and high thyroid stimulating hormone levels in children with Down's syndrome. J Pediatr Endocrinol Metab 2002; 15 (9): 1543-8.

15.- Sanz J: Síndrome de Down e hipertiroidismo: comunicación de 3 casos. Rev Méd Chile 1999; 127 (7): 967-9.

16.- Bader AA, August GP, Austin A: Hypertransaminasemia in two children with hyperthyroidism. J Pediatr Gastroenterol Nutr 2001; 32: 484-6.

17.- Cooper DS: Antithyroid drugs. N Engl J Med 2005; 352 (9): 905-17.

18.- Rivkees $S$ : Editorial: radioactive iodine use in childhood Graves' disease: time to wake up and smell the i-131. J Clin Endocrinol Metab 2004; 89 (9): 4227-8.

19.- Sherman J, Thompson G, Lteif A, et al: Surgical management of Graves disease in childhood and adolescence: an institutional experience. Surgery 2006; 140 (6): 1056-62. 